\title{
Online milk composition analysis with an on-farm near- infrared sensor
}

\section{Authors}

4 Jose A. Diaz-Olivares ${ }^{1 a}$, Ines Adriaens ${ }^{\text {ab }}$, Els Stevens ${ }^{c}$, Wouter Saeys ${ }^{b}$, Ben Aernouts ${ }^{a}$

$5{ }^{a}$ KU Leuven, Department of Biosystems, Biosystems Technology Cluster, Campus Geel, Kleinhoefstraat 4,

62440 Geel, Belgium

7 b KU Leuven, Department of Biosystems, Division Mechatronics, Biostatistics and Sensors, Kasteelpark

8 Arenberg 30, 3001 Leuven, Belgium

9 c Province of Antwerp, Hooibeekhoeve, Hooibeeksedijk 1, 2440 Geel, Belgium

$10{ }^{1}$ Corresponding author: Jose A. Diaz-Olivares, KU Leuven, Department of Biosystems, Biosystems

11 Technology Cluster, Campus Geel, Kleinhoefstraat 4, 2440 Geel, Belgium, +32 (0)14 721467 ,

12 jose.diaz@kuleuven.be

14 Abstract

15 On-farm monitoring of milk composition can support close control of the udder and metabolic health of

16 individual dairy cows. In previous studies, near-infrared (NIR) spectroscopy applied to milk analysis has

17 proven useful for predicting the main components of raw milk (fat, protein, and lactose). In this

18 contribution, we present and evaluate a precise tool for online milk composition analysis on the farm. For

19 each milking, the online analyzer automatically collects and analyses a representative milk sample. The

20 system acquires the NIR transmission spectra of the milk samples in the wavelength range from 960 to

$21 \quad 1690 \mathrm{~nm}$ and performs a milk composition prediction afterward.

22 Over a testing period of 8 weeks, the sensor collected 1165 NIR transmittance spectra of raw milk samples

23 originating from 36 cows for which reference analyses were performed for fat, protein, and lactose. For

24 the same online sensor system, two calibration scenarios were evaluated: training post-hoc prediction 
period, with different cows in the calibration and test set, and training real-time prediction models

27 exclusively on the samples acquired in the first week of the testing period $(n=308)$.

28 The obtained prediction models were thoroughly tested on all the remaining samples not included in the

29 calibration sets ( $n$ respectively 846 and 857 ). For the post-hoc prediction models, this resulted in an overall

30 prediction error (root-mean-squared error of prediction, RMSEP) smaller than $0.080 \%$ (all \% are in wt/wt)

31 for milk fat (range 1.5-6.3\%), protein (2.6-4.3\%) and lactose (4-5.1\%), with a coefficient of determination

$32 \mathrm{R}^{2}$ of $0.989,0.947$ and 0.689 for fat, protein, and lactose respectively. For the real-time prediction models,

33 the RMSEP was smaller than $0.092 \%$ for milk fat and lactose, and $0.110 \%$ for protein, with an $\mathrm{R}^{2}$ of 0.989

34 (fat), 0.894 (protein) and 0.644 (lactose). The milk lactose predictions could be further improved (RMSEP

$\left.35=0.088 \%, R^{2}=0.675\right)$ by taking into account a cow-specific bias. The presented online sensor system using

36 the real-time prediction approach can thus be used for detailed and autonomous on-farm monitoring of

37 milk composition after each individual milking, as its accuracy is well within the requirements by the

38 International Committee for Animal Recording (ICAR) for on-farm milk analyzers and even meet the

39 standards for laboratory analysis systems for fat and lactose. For this real-time prediction approach, a drift

40 was observed in the predictions, especially for protein. Therefore, further research on the development

41 of online calibration maintenance techniques is required to correct for this model drift and further improve

42 the performance of this sensor system.

\section{Keywords}

44 near-infrared spectroscopy; milk; health monitoring; real-time prediction;

\section{Introduction}

46 As the metabolism of dairy cows is heavily conditioned towards milk production, the milk composition can

47 inform about the cow's nutritional, metabolic, and health status (McParland et al., 2014). In standard dairy 
practices, cows are milked twice or three times a day, which implies that milk samples can be taken and

49 analyzed regularly without interfering in the animal's daily life. Therefore, frequent analysis of the

50 produced milk can be considered a very efficient way to monitor the performance, efficiency, and welfare

51 of individual dairy cows.

52 Critical dairy health issues such as ketosis and mastitis produce changes in the concentration of the main

53 components of raw milk (fat, protein, and lactose). For example, mastitis causes a decrease in lactose

54 concentration (Bobbo et al., 2016; Forsbäck et al., 2010, 2009; Gonçalves et al., 2016), while ketosis is

55 associated with increased fat and decreased protein contents (Brandt et al., 2010). Frequent and accurate

56 monitoring of these changes over time allows for detecting alterations of the udder and metabolic health

57 of individual cows (Mäntysaari et al., 2019).

58 Visible (Vis) and near-infrared (NIR) spectroscopy is a promising technique for the on-farm monitoring of

59 the main components of raw milk, a potential scrutinized by numerous researchers in the past (Kawamura

60 et al., 2007; Kawasaki et al., 2008; Melfsen et al., 2012; Tsenkova et al., 1999). Several commercially

61 available technologies make use of this technique, mainly in the Vis and short-wave NIR wavelength range

62 (400 to $1000 \mathrm{~nm}$ ). Despite the low cost of detectors for that wavelength range, the performance in this

63 region often provides unsatisfactory results for the prediction of milk composition (Fadul-Pacheco et al.,

64 2018; Kaniyamattam and De Vries, 2014). This suboptimal performance is apparent when compared to

65 NIR analyses performed using the long-wave NIR (LW-NIR) range from 1000 to $1700 \mathrm{~nm}$ (Aernouts et al.,

66 2011).

67 Both reflectance and transmittance spectra can be collected using LW-NIR spectroscopy. In previous

68 works, LW-NIR transmittance has shown an exceptional performance for raw milk composition prediction,

69 with a root-mean-square error (RMSE) lower than $0.13 \%(w t / w t)$ and a coefficient of determination $\left(R^{2}\right)$ 
70 higher than 0.9 for fat, protein and lactose predictions (Aernouts et al., 2011; Saranwong and Kawano,

71 2008; Tsenkova et al., 1999).

72 Using LW-NIR reflectance, fat and protein show a strong prediction performance, as fat globules and casein 73 micelles are strongly linked to the NIR scattering. These scattering phenomena are caused by their physical 74 structures, with the extensive variation in size and number of fat globules and casein micelles affecting the 75 variation in light scattering (Postelmans et al., 2020), in addition to the absorbance of NIR radiation by 76 their covalent bonds (Bogomolov and Melenteva, 2013). In contrast, lactose predictions are relatively poor 77 (RMSE higher than $0.15 \%$ and an $\mathrm{R}^{2}$ lower than 0.75 ), as the majority of the reflected photons acquired by 78 the sensor have limited interaction with the milk serum, in which lactose is diluted (Aernouts et al., 2015b).

79 Despite the potential of LW-NIR spectroscopy shown in previous studies, the strong absorption of LW-NIR

80 radiation by water molecules and the severe light scattering by fat globules and casein micelles pose 81 significant challenges for its on-farm use. For example, the thickness of the sample can only be 1 to $2 \mathrm{~mm}$ 82 in order to obtain sufficient LW-NIR transmittance (Aernouts et al., 2011; Tsenkova et al., 1999), which 83 complicates its in-line implementation due to its impact on the collected milk flow. This implies that a 84 representative milk sample needs to be taken using a bypass, as demonstrated by Kawasaki et al. 85 (Kawasaki et al., 2008).

86 Although many researchers have already shown the potential of LW-NIR spectroscopy for milk

87 composition analysis in the laboratory, studies testing this technology under farm conditions are scarce 88 and often include only a limited number of milk samples measured over a few test days (Kawasaki et al., 89 2008; Melfsen et al., 2012). Moreover, all previous studies, even the ones performed on farms, have made 90 use of a post-hoc approach to predict the milk composition, in which the samples to train and test the 91 prediction models were posteriorly selected from the complete dataset, both covering the full variability 92 and time span of that dataset. None of these previous studies have considered a real-time prediction of 
93 the milk composition using calibration models that were already established before a set of validation

94 samples was measured.

95 In this study, we present an on-farm sensor system making use of LW-NIR spectroscopy to monitor the

96 milk composition for each individual cow and every milking session. The main advantage of this system

97 against currently established milk composition analysis methods is the possibility to install it in an AMS

98 (automated milking system) allowing to perform milk composition predictions after every milking, in a fast,

99 cost-efficient, and non-destructive manner. The goal of this work is to evaluate the accuracy of this tool

100 and its potential for online measurement of the milk fat, protein, and lactose concentrations, with a real-

101 time prediction approach. Moreover, to investigate model drift, the real-time predictions are compared

102 to the ones obtained with the post-hoc approach followed in former studies. Additionally, it was

103 investigated whether a cow-specific bias correction can further improve the performance of the real-time

104 prediction models.

\section{Materials and methods}

\subsection{Sensor system to measure the long-wave near-infrared transmittance of raw milk}

The sensor system, illustrated in Figure 1, consists of (a) a NIR spectrometer (1.7-256 Plane Grating

110256 pixels (2.86 $\mathrm{nm}$ pixel resolution in the 960 to $1690 \mathrm{~nm}$ range); (b) a 20 Watt halogen light source

111 (998079-14, Welch Allyn, New York, USA); (c) an optical measuring unit and (d) a dedicated computer

112 which runs the control and spectral acquisition software in LabView 2012 (National Instruments, Austin,

113 USA). All previous elements are contained in a compact and temperature-controlled (PowerCool, Laird,

114 Liberec, Czech Republic) steel housing, which protects them from ammonia, dust, and moisture. 
The optical measuring unit comprises (c.1) a round borosilicate cuvette with an inner thickness and diameter of respectively 2 and $26 \mathrm{~mm}$ and containing $1.2 \mathrm{~mL}$ of raw milk during spectral sample measurements; a spectral reference pair with a (c.2) $2 \mathrm{~mm}$ thick Spectralon (Labsphere, North Sutton, USA) white standard and a (c.3) 1\% Acktar Black (Acktar, Hohenaspe, Germany) coated plate as a dark standard, and (c.4) a translation stage, consisting of a filter slide (FS40, OWIS, Frankfurt, Germany) which was automated with a stepper motor (L4118L1804, Nanotec, Munich, Germany). This translation stage holds the cuvette and the spectral reference pair and allows to alternate between them in order to perform spectral reference measurements before measuring the milk sample. The transmitted light is collected by a converging-type lens with a focal length of $31.6 \mathrm{~mm}$ (770303-9020-000 2:1, Carl Zeiss, Jena, Germany) and guided to the spectrometer by an optical fiber ( $600 \mu \mathrm{m}$ core diameter, low-OH). The lens is aligned with the center of the halogen light source and the translation stage moves perpendicularly to the lens-halogen axis when alternating between the cuvette and the white and dark spectral references. When the dark spectral reference is in position, the stray light and background noise of the spectrometer are quantified.

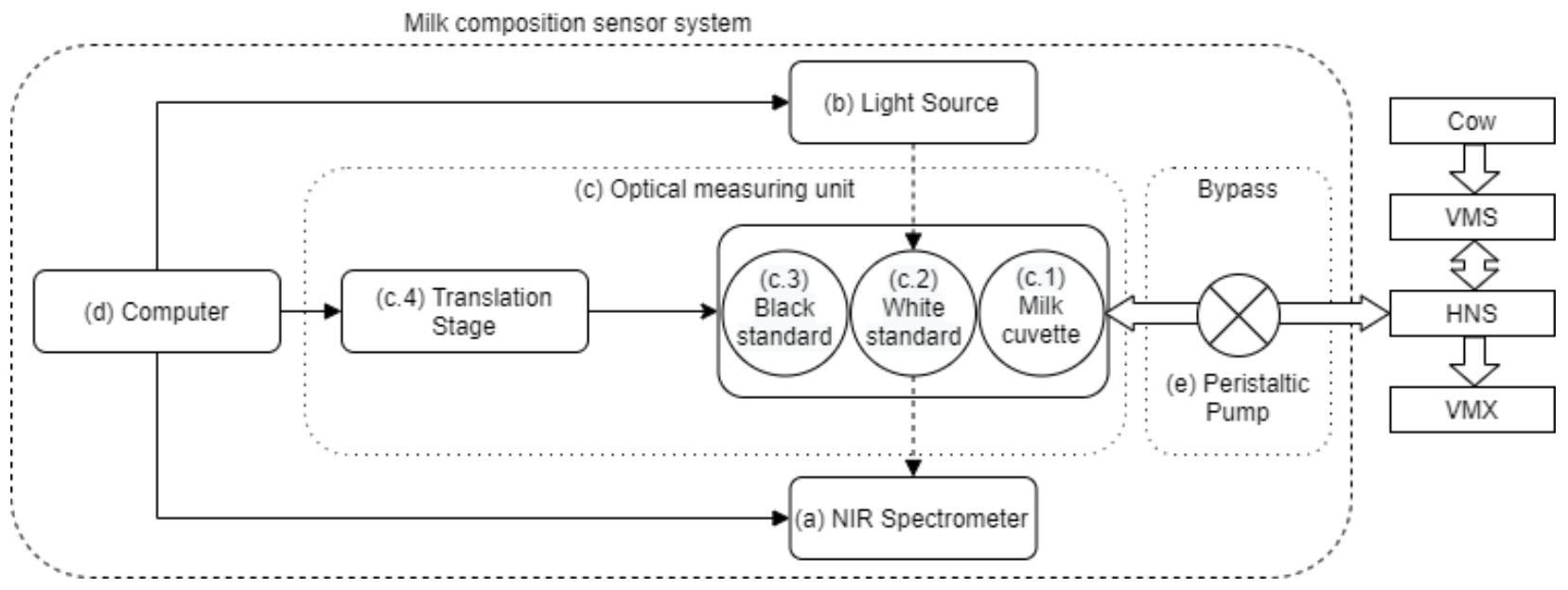


133 For each milk sample in the cuvette, the NIR spectrometer recorded LW-NIR spectra in transmittance mode

134 in the wavelength range from 960 to $1690 \mathrm{~nm}$. To obtain a high signal-to-noise ratio, these spectra were 135 acquired with an integration time of 100 ms collecting 100 repeated measurements per milk sample,

136 aggregated into an average spectrum for each sample. In addition, dark and white reference spectra were 137 acquired using the same settings while the milk sample was loaded in the cuvette, just before performing 138 a spectral recording for that milk sample.

\subsection{Experimental setup and reference analysis of milk samples}

140 The sensor system was tested in combination with a VMS ${ }^{\mathrm{TM}}$ Classic (DeLaval, Tumba, Sweden) AMS at the 141 experimental dairy farm 'Hooibeekhoeve' of the province of Antwerp (Geel, Belgium). A Herd Navigator ${ }^{\mathrm{TM}}$ 142 sampler (HNS Supra+, DeLaval) was installed on the AMS collecting a $300 \mathrm{~mL}$ sample that is representative 143 of all the milk collected during that milking (ICAR, 2017a). With this sampler, a fraction ( $\pm 100 \mathrm{~mL})$ of the 144 collected milk samples can be pumped to the Herd Navigator ${ }^{\mathrm{TM}}$ analyzer (DeLaval) for milk biomarker 145 analysis, while another fraction $\left( \pm 30 \mathrm{~mL}\right.$ ) can be sent to the $\mathrm{VMX}^{\mathrm{TM}}$ (DeLaval) to take a milk sample for 146 laboratory milk analysis in the context of milk recording programs. The sensor system was installed as a 147 bypass on the backflow of the Herd Navigator ${ }^{\mathrm{TM}}$ sampler and received at least $150 \mathrm{~mL}$ of raw milk. A 148 peristaltic pump (EZ OEM Pump, Verderflex) created a controlled milk flow from the bypass to the cuvette

149 (Figure 1e) and evacuated the milk once the spectral analysis was performed. The temperature of the 150 sample in the cuvette was not monitored nor regulated. Thanks to thorough sample mixing in the Herd 151 Navigator ${ }^{\mathrm{TM}}$ sampler, the composition of the milk analyzed by the sensor system and the milk taken by the $152 \mathrm{VMX}^{\mathrm{TM}}$ milk sampler can be assumed identical. Cleaning of the milk circuit and cuvette was performed by 153 allowing the flush of an alkaline detergent (UltraClean ${ }^{\mathrm{TM}}$, DeLaval) through the bypass, during the 154 automatic cleaning process of the $\mathrm{VMS}^{\mathrm{TM}}$ Classic and Herd Navigator ${ }^{\mathrm{TM}}$ sampler. 
Over a period of eight weeks, the sensor system analyzed raw milk originating from 41 Holstein cows

156 (lactation stage $168 \pm 84$ days in milk, parity $2.0 \pm 1.1$ ). On average, cows were milked 2.6 times a day.

157 However, spectral analyses were only performed when a milk sample was taken for reference analysis in

158 the context of an unrelated feeding trial. The periods for which milk samples were collected varied 159 between 21 and 85 hours of non-interrupted measurements per week, with an average of 158 samples

160 taken per week. The milk samples taken by the $\mathrm{VMX}{ }^{\mathrm{TM}}$ were preserved with bronopol $(0.3 \mathrm{mg} / \mathrm{mL})$ and 161 analyzed at the Milk Control Center (MCC Vlaanderen, Lier, Belgium) within three days after sample 162 collection. In this experiment, the reference analysis (fat, protein, and lactose) of each sample was 163 performed according to ISO 9622 (ISO, 2013) with the Milkoscan FT+ (Foss A/S, Hillerød, Denmark), which 164 has an accuracy $<1.0 \%$ coefficient of variation (CV) for fat and $<0.9 \% \mathrm{CV}$ for protein and lactose. For this 165 reference analysis, based on the prediction of milk composition by the use of Fourier Transform mid166 infrared spectroscopy, short-term stability tests are performed routinely.

167 In total, milk samples and spectral measurements from 1270 individual milkings were collected during the 168 experimental period, corresponding to approximately $22 \%$ of the number of milkings $(n=5969)$ in that 169 eight-week period. Fifty-nine of them originated from four dairy cows that calved during the trial and from 170 one individual animal that was less than five days in milk at the beginning of the experiment. To avoid the 171 inclusion of colostrum samples, they were removed from the dataset (Abd El -Fattah et al., 2012). Forty172 six additional samples had to be discarded as a consequence of unsuccessful reference analyses, caused 173 by a failure of the $\mathrm{VMX}^{\mathrm{TM}}$ on filling sample tubes with sufficient milk. The final dataset consisted of 1165 174 raw milk samples from 36 cows for which both transmission spectra and reference analyses were available. 
176 The dataset with transmittance spectra and the results from the reference analyses were imported in R

177 version 3.5.1 (R Core Team, 2018) to be analyzed with a custom chemometrics toolbox (Aernouts et al., 178 2020).

179 By default, a pair of dark and white reference spectra was acquired every time a milk sample spectrum 180 was recorded. This pair of reference spectra was used to normalize the sample spectra according to the

181 formula Transmittance spectrum $=\frac{\text { sample spectrum }- \text { dark spectrum }}{\text { white spectrum }- \text { dark spectrum }}$. This procedure corrects the 182 spectrum of each sample for drift in the spectral sensitivity of the spectrometer and the intensity spectrum 183 of the light source, both mainly influenced by stray light or environmental temperature changes (Andersen 184 et al., 2013).

185 This correction is only needed when the drift effect is higher than the stochastic noise introduced by the 186 acquisition of the reference spectra (Slutsky, 1997). Therefore, to identify degradation in the prediction 187 caused by this drift and accordingly improve the milk composition predictions, different time intervals for 188 acquiring a new pair of dark and white reference spectra were evaluated. Based on this analysis, a new 189 spectral reference pair was considered right after connecting the sensor system to the AMS and every 0.5 190 hours after this initialization.

\subsection{Development and validation of the post-hoc prediction models}

192 In previous studies, a post-hoc prediction approach was followed in which the samples to train and test 193 the prediction models were selected from a single dataset, both covering the full variability and time span 194 of that dataset. For an objective comparison, a similar approach was initially followed in this study too. To 195 this end, the duplex algorithm (Snee, 1977) was used to divide the total dataset of the milk transmittance 196 spectra and their respective reference analyses into an elaborate and representative calibration set of 197 approximately 300 samples (Shetty et al., 2011) and a test set containing the remainder of the samples. 
198 Not taking into account the time when the samples were measured, the post-hoc approach uses the 199 Mahalanobis distance between the samples in the 3-dimensional space of the reference analyses (fat, 200 protein, and lactose) to obtain two subsets with similar descriptive statistics and correlations for these 201 milk components. To prevent over-optimistic test results due to modeled cow-specific effects, all samples 202 from the same cow were grouped either in the calibration or test set (Kemps et al., 2010), with 9 cows in 203 the calibration set and 27 cows in the validation set.

204 The wavelength range in the spectra where the absorption by water molecules is predominant (1360 to $2051500 \mathrm{~nm}$ ), and thus the transmittance signals are very low, were removed to reduce the introduction of 206 spectral noise. The pre-processing applied to the spectra was a combination of five steps. Initially, either 207 a logarithmic spectral transformation step or no transformation was applied, followed by a second step 208 with either multiplicative scatter correction, standard normal variates weighting, detrending, baseline 209 correction, or no correction. As a third step, either no derivative or a first or second-order Savitzky-Golay 210 derivative with a second-order polynomial filter and with 10 different spectral window lengths was 211 applied, followed by a fourth step in which interference removal by orthogonal signal correction (OSC) was 212 tested. As a final step, mean-centering was performed. These five steps resulted in 420 possible pre213 processing combinations. For each of these combinations, the transmittance spectra of the calibration 214 samples were used to train their respective partial least squares regression (PLSR) models to predict the 215 individual milk components. Next, also four different variable selection methods (variable importance in 216 projection, jack-knife, reversed interval PLSR, and forward interval PLSR) were applied on the pre217 processed NIR spectra to obtain, each resulting in a set of most relevant wavebands. The maximum 218 number of latent variables for the PLSR model was 20. The performances of these 4 PLSR models were 219 compared mutually and with the model that uses all wavebands. The optimal number of latent variables 220 for each PLSR model, the best spectral preprocessing, and the most informative spectral wavelengths were 221 selected based on the model performances in group-wise cross-validation for the samples in the 
222 calibration set (Aernouts et al., 2020). To this end, the calibration set was split into ten groups, each 223 containing all the spectra of a single cow. Accordingly, all samples from the same cow were either included 224 or excluded from the training set to prevent modeling cow-specific effects or relations (Kemps et al., 2010).

225 The least complex model (with the lowest number of latent variables) that did not perform significantly 226 worse than the model with the lowest root-mean-squared error of cross-validation (RMSECV) was selected 227 (Haaland and Thomas, 1988). This comparison was based on one-sided paired t-tests $(a=0.05)$ applied to 228 the absolute residuals of the cross-validated calibration samples (Cederkvist et al., 2005). This procedure was repeated for the three different milk components, obtaining a prediction model per component. As

230 the resulting models were trained on samples extracted from the entire experimental period of 8 weeks, 231 they are hereafter referred to as the post-hoc prediction models. Using these models, milk composition 232 predictions were obtained for the samples in the test set, and the respective residuals were derived. These 233 errors were then combined into a root-mean-square error of prediction (RMSEP).

\subsection{Development and validation of the real-time prediction models}

235 To evaluate the performance of the sensor system in predicting milk composition after each individual 236 milking, which is the main goal of this study, prediction models for fat, protein, and lactose were trained 237 on samples taken in the first week only $(n=308)$ and validated on all subsequent samples, which were 238 measured over the following seven weeks $(n=857)$. In contrast with the post-hoc approach, the same 239 cows are present in the calibration and test sets. These models are hereafter referred to as the real-time 240 prediction models. The procedures to train, test, and compare the models were identical to the ones 241 previously described for the post-hoc prediction models.

242 Finally, to compare the prediction performance of the real-time and the post-hoc approaches, they were 243 contrasted using a statistical test on the squared residuals of the milk component predictions for the 
245 the two approaches were compared with an unpaired t-test, in order to assess if there was a significant

246 difference $(a=0.05)$ between them.

2472.6 Analysis of the cow-specific bias on the milk component predictions by the real-time

$248 \quad$ models

249 To evaluate whether bias was present in the milk composition predictions for specific dairy cows, an

250 analysis of this phenomenon was undertaken (Anderson et al., 2016). Over time, this bias can be observed

251 as a cow-specific baseline in the residuals that are obtained after subtracting the predicted milk 252 compositions from the respective reference analyses. For the real-time models, all cows were represented

253 in the calibration set, as well as the test set, which allows the estimation of each individual cow-specific

254 bias in the calibration set, and to apply it in the test set. This was not true for the post-hoc models, due to

255 how the duplex procedure was implemented to split the original dataset. Accordingly, the cow-specific

256 bias on the three milk component predictions was only studied for the real-time models.

257 The median value of the residuals of the samples in the calibration set was calculated for each individual 258 cow, as its cow-specific bias. First, the agreement between those medians and the prediction residuals of 259 the samples in the test set was studied for all cows using the concordance correlation coefficient (Lin, 260 1989). Additionally, these cow-specific biases obtained from the calibration set was then subtracted from 261 all further milk composition predictions for that same animal in the test set. Next, the performance of the 262 real-time model for each component was evaluated before and after the correction of the cow-specific 263 bias. This comparison was performed with a paired t-test on the squared residuals of the samples in the 264 test set, in order to assess if there was a significant difference $(a=0.05)$ between them. 


\section{Results and discussion}

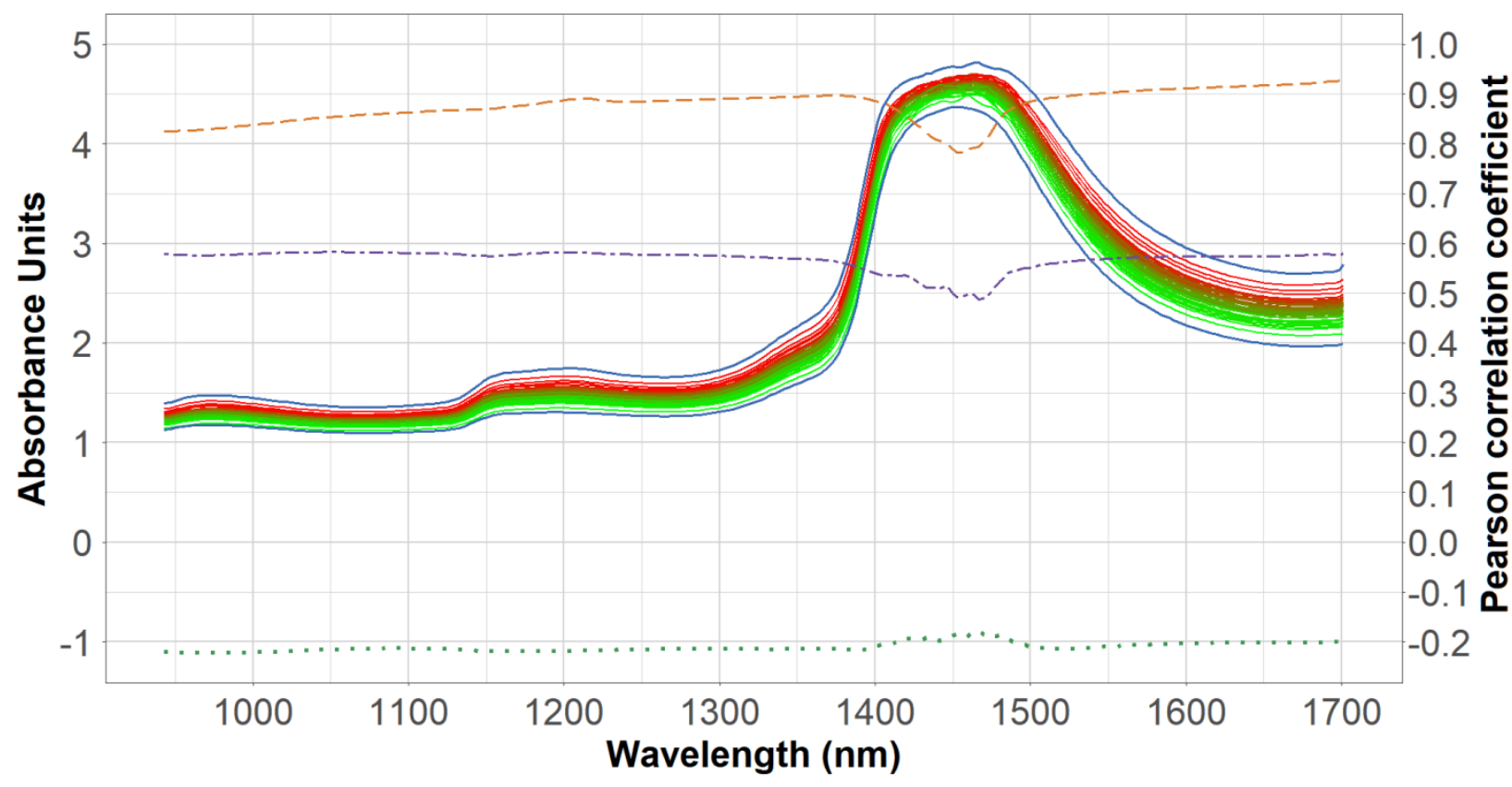

Figure 2. Absorbance spectra for a single dairy cow (solid green/red gradient lines) collected over a period of 8 weeks. Green represents lower fat values, while red symbolizes higher fat values, for a fat range of $2.27 \%-5.48 \%$ for this cow. Minimum and maximum (bold blue solid lines) absorbance spectral values for the entire dataset are depicted. The Pearson correlation coefficients illustrate for every wavelength the linear correlation between the near-infrared absorbance by the 1165 raw milk samples and their fat (orange dashed line), protein (purple dot-dashed line), and lactose (green dotted line) concentration.

In Figure 2, the absorbance spectra, obtained by the logarithmic transformation of the inverse of the transmittance spectra, is illustrated as solid blue lines for all the milk samples of a single dairy cow. It presents the variability in the observations acquired from the same animal across the duration of the blue lines. Clear absorbance peaks can be observed around 970, 1200 and $1450 \mathrm{~nm}$. The absorbance units between 4 and 5 for the latter peak indicate that only a very small fraction $\left(10^{-4}-10^{-5}\right)$ of the incident photons around this wavelength range was transmitted, resulting in a low signal to noise ratio. 
281 The high correlation $(r>0.78)$ between the fat content and the absorbance values over the full wavelength 282 range can be attributed to the scattering of light by the fat globules. As a higher fat content results in 283 stronger light scattering, it reduces the transmittance and thus increases the absorbance, resulting in a 284 nearly constant correlation between the fat content and the NIR absorbance over the full wavelength 285 range. In Figure 2, the maximum and minimum absorbance spectra (bold blue solid lines) of the entire 286 dataset corresponded to respectively the maximum (6.25\%) and the minimum fat percentage (1.54\%). An 287 absorption band of $\mathrm{C}-\mathrm{H}$ bond overtone, characteristic of fat molecules, can be observed as a slight increase 288 in the correlation with the fat content of the NIR absorption around $1210 \mathrm{~nm}$ (Zamora-Rojas et al., 2013). 289 A significant positive correlation ( $r=0.49$ to 0.59$)$ between the absorbance values and the protein content 290 can be observed. This relationship can be mainly attributed to the typical correlation between the protein 291 and the fat content of milk (Rosati and Van Vleck, 2002). In contrast, a very weak negative correlation ( $r=$ $292-0.19$ to -0.23$)$ was found between milk lactose content and the NIR absorption spectra. Protein and 293 lactose do not display clear absorption peaks, as they overlap with the more dominant absorption of water 294 and fat.

\subsection{Development and validation of the post-hoc prediction models}

297 Following the duplex algorithm, the original dataset of 1165 raw milk samples was split between a 298 calibration set $(n=319)$ and a test set $(n=846)$. The descriptive statistics of the fat, protein, and lactose 299 content of the resulting sets and their correlations are summarized in Table 1. The comparable average 300 and variability show that the data splits are representative for the whole dataset, which is critical for 301 developing a robust prediction model (Saeys et al., 2008). Comparing the statistical parameters of this 302 dataset to the data from milk recording programs in Flanders (Aernouts et al., 2011), showed that our 303 samples also have comparable values and variability as the cow population of this region. 
Table 1. Basic statistics and Pearson correlations for the main milk components in the calibration and test sets used to construct the post-hoc prediction models.

\begin{tabular}{|c|c|c|c|c|c|c|c|c|c|c|c|c|}
\hline \multirow{3}{*}{ Component } & \multicolumn{6}{|c|}{ Calibration $(n=319)$} & \multicolumn{6}{|c|}{ Test $(n=846)$} \\
\hline & \multicolumn{4}{|c|}{ Basic statistics (\% wt/wt) } & \multicolumn{2}{|c|}{ Pearson corr. } & \multicolumn{4}{|c|}{ Basic statistics (\% wt/wt) } & \multicolumn{2}{|c|}{ Pearson corr. } \\
\hline & Mean & SD & Min & Max & Fat & Protein & Mean & SD & Min & Max & Fat & Protein \\
\hline Fat & 3.54 & 0.92 & 1.54 & 5.81 & 1 & - & 3.5 & 0.76 & 1.54 & 6.25 & 1 & - \\
\hline Protein & 3.44 & 0.34 & 2.75 & 4.26 & 0.51 & 1 & 3.42 & 0.34 & 2.63 & 4.34 & 0.57 & 1 \\
\hline Lactose & 4.65 & 0.19 & 3.98 & 5.1 & -0.11 & -0.23 & 4.71 & 0.14 & 3.99 & 5.03 & -0.19 & -0.11 \\
\hline
\end{tabular}

Different time intervals were evaluated for acquiring a new pair of dark and white references. Based on this analysis, it was decided to take a new spectral reference pair at the start of each session after 310 connecting the sensor system to the AMS and every 0.5 hours after this initialization. Based on the 311 obtained results, it is expected that if new spectral references are taken with a higher frequency than this 312 optimum, unnecessary stochastic noise, typical for spectral measurement, would be introduced. In 313 contrast, if new spectral references are taken with a lower frequency than this optimum, significant drift 314 in the spectral output of the light source and spectral sensitivity of the spectrometers might not be fully 315 captured and accounted for, also negatively affecting the results. 
(a)

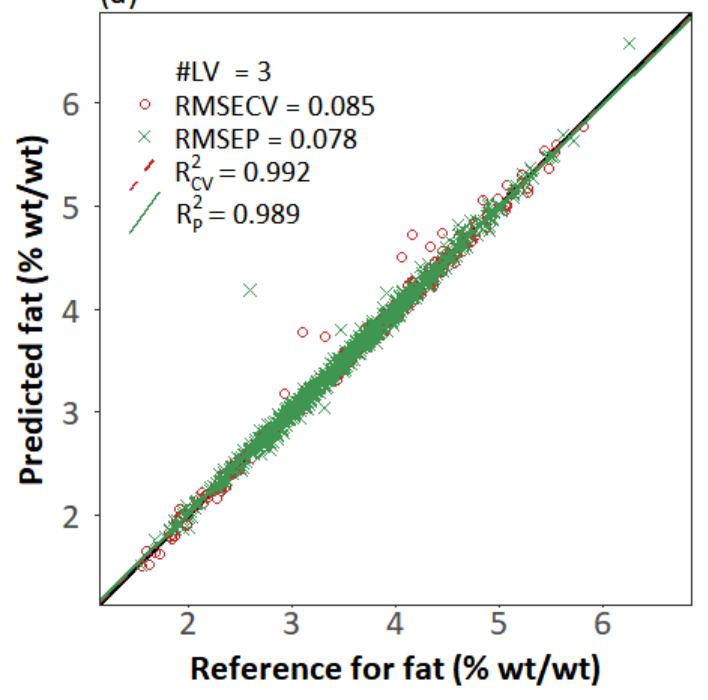

(b)

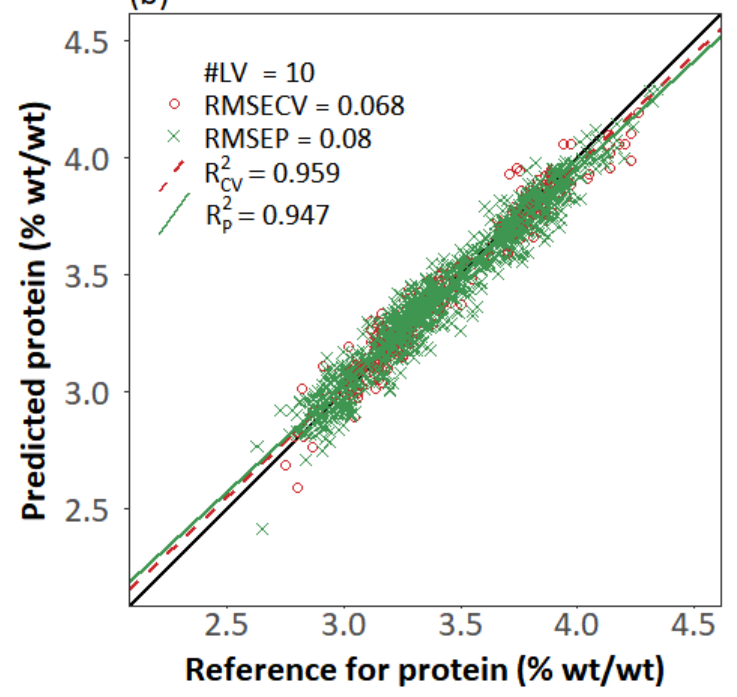

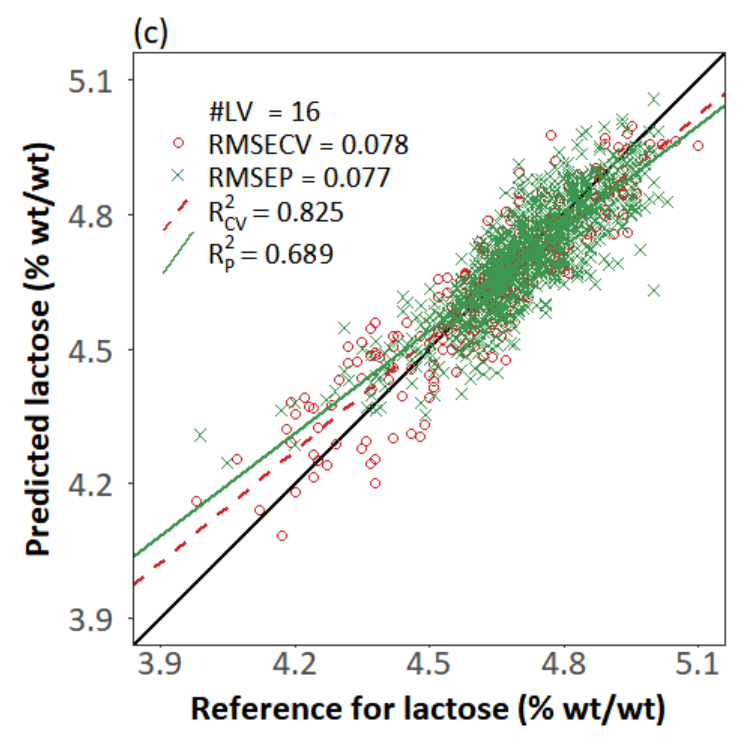

318 Figure 3. Scatter plots of the predicted versus reference milk composition content for the calibration set in cross-validation (red 319 circles) and the test set (green crosses) for (a) fat, (b) protein, and (c) lactose, for the post-hoc approach. \#LV = number of latent 320 variables used by the model; RMSECV = root-mean-square error of cross-validation; RMSEP = root-mean-square error of 321 prediction; $R_{C V}^{2}=$ coefficient of determination for cross-validation; $R_{P}^{2}=$ coefficient of determination for prediction. The black solid 322 lines are the identity lines (1:1 correlation line). The pre-processing and variable selection applied is detailed in Table 4.

323 Figure 3 shows the prediction performance of the post-hoc models for the calibration samples in crossvalidation (red circles) and the test samples (green crosses). Very good predictions are obtained for all three milk components, with RMSEP values smaller than $0.08 \%$ (wt/wt). For fat and protein, more than 
$98 \%$ and $94 \%$ of the variation in the reference analyses for these components can be explained by their

327 respective prediction model. The lower $R^{2}$ values observed for lactose $\left(R_{\mathrm{CV}}^{2}=0.825\right.$ and $\left.R_{\mathrm{P}}^{2}=0.689\right)$ can be 328 partly attributed to the limited range $(3.98 \%-5.1 \%(w t / w t))$ of this component in this particular dataset.

329 Additionally, it can be observed (Figure 3c) that the majority of the test samples have a lactose 330 concentration greater than $4.5 \%(w t / w t)$, while lactose calibration samples span a wider distribution, 331 contributing to the discrepancy between the $R_{\mathrm{CV}}^{2}$ and $R_{\mathrm{P}}^{2}$ values while the RMSE values are very similar. 332 This suggests that the split performed by the duplex algorithm had limited success for lactose. For both fat 333 and lactose, RMSECV values are slightly higher than RMSEP, which can be partly attributed to the greater 334 dispersion of the calibration data for both milk components (SD of $0.92 \%$ for fat and $0.19 \%$ for lactose) 335 against the test data (SD of $0.76 \%$ for fat and $0.14 \%$ for lactose, all $\% w t / w t$ ) and the higher presence of 336 samples that are further away from the identity line (Figure 3) in the calibration data compared to the test 337 data. The PLSR model for lactose had the highest complexity (16 latent variables), which is considerably 338 higher than the 3 and 10 latent variables for fat and protein, respectively.

339 As can be seen in Table 2, the performance of these models is comparable to the results for the prediction 340 of the raw milk composition based on LW-NIR spectra reported by other researchers. The selection of 341 these works prioritized potential on-farm applications and the use of the LW-NIR wavelength range 342 (Aernouts et al., 2011; Kawasaki et al., 2008; Melfsen et al., 2012; Saranwong and Kawano, 2008; Tsenkova 343 et al., 2001). These studies were all performed in transmission mode, except for Melfsen et al., (2012), 344 who used diffuse reflectance mode.

345 Table 2. Comparison of obtained model performances to the values reported by other researchers.

\begin{tabular}{|c|c|c|c|c|c|c|c|}
\hline \multirow{2}{*}{ Source } & \multirow{2}{*}{$n$} & \multicolumn{3}{|c|}{ RMSEP (\% wt/wt) } & \multicolumn{3}{|c|}{$R_{\mathrm{P}}^{2}$} \\
\hline & & Fat & Protein & Lactose & Fat & Protein & Lactose \\
\hline Post-hoc model & 846 & 0.078 & 0.08 & 0.077 & 0.989 & 0.947 & 0.689 \\
\hline Kawasaki et al., 2008 & 72 & $0.255^{\Delta}$ & $0.149^{\Delta}$ & $0.258^{\Delta}$ & 0.95 & 0.72 & 0.83 \\
\hline
\end{tabular}


Melfsen et al., 2012*

Tsenkova et al., 2001

Aernouts et al., 2011

Saranwong and Kawano, 2008

$\begin{array}{ccccccc}262 & 0.09^{\Delta} & 0.05^{\Delta} & 0.06^{\Delta} & 0.998 & 0.98 & 0.92 \\ 86 & 0.258^{\Delta} & 0.13^{\Delta} & 0.084^{\Delta} & 0.98 & 0.45 & 0.72 \\ 100 & 0.046 & 0.133 & 0.115 & 0.997 & 0.927 & 0.883 \\ & & & & & & \\ 47-43-46 & 0.03^{\Delta} & 0.07^{\Delta} & 0.094^{\Delta} & 0.99 & 0.96 & 0.89\end{array}$

RMSEP = Root-mean-square error of prediction; $R_{\mathrm{P}}^{2}=$ coefficient of determination; $n=$ number of samples in the test set.

${ }^{\Delta}$ RMSEP values calculated from standard error of prediction (SEP), bias, and number of samples, provided by the authors, with the following relationship: $\operatorname{RMSEP}=\sqrt{((n-1) / n) \times S E P^{2}+b i a s^{2}}$.

*In this study, diffuse reflectance was used, all other studies used transmittance.

${ }^{\text {r} S a r a n w o n g ~ a n d ~ K a w a n o ~(2008) ~ u s e d ~ a ~ d i f f e r e n t ~ n u m b e r ~ o f ~ t e s t ~ s a m p l e s ~ f o r ~ f a t, ~ p r o t e i n, ~ a n d ~ l a c t o s e, ~ r e s p e c t i v e l y . ~}$

RMSEP and $R_{\mathrm{P}}^{2}$ values were considered in the examination of the literature sources (Table 2). The analysis by Saranwong and Kawano (2008), Aernouts et al. (2011), and the post-hoc model achieved outstanding accuracy for fat prediction, with RMSEP values smaller than 0.08\% (wt/wt). Saranwong and Kawano (2008), Melfsen et al. (2012) and the post-hoc approach of the present study are the best performing for predicting protein content, with RMSEP values that did not exceed $0.08 \%$ (wt/wt). Melfsen et al. (2012) and the posthoc model in this study are very good for predicting lactose content in milk, with RMSEP values under 0.08 $\%(w t / w t)$.

In comparison to the results reported by other researchers, in general, we obtained low RMSEP and high $R_{\mathrm{P}}^{2}$ values, underlining the general suitability of the sensor system and calibration procedure for predicting the main constituents in raw milk.

However, by selecting calibration samples throughout the experiment, the literature sources and present post-hoc models do not provide an objective and complete image on how the system would perform in a real-time setting. In order to evaluate the capability of the on-farm sensor system to perform real-time predictions, the validation should be performed on samples that are measured after the initial calibration, thus being date-independent of the samples used for training the prediction models. 
To test the potential for real-time prediction of the milk composition, the 308 raw milk samples collected

368 in the first week of the trial were assigned to the calibration set, while those collected in weeks 2 to 8 ( $n=$

369 857) were assigned to the test set. The descriptive statistics for the fat, protein, and lactose content of

370 both sets and their correlations are summarized in Table 3. This shows that the samples of the first week

371 are representative of the variation present in the samples of weeks 2 to 8.

Table 3. Descriptive statistics and Pearson correlations of the main milk components in the calibration and test set for the real-time 374 prediction models.

\begin{tabular}{|c|c|c|c|c|c|c|c|c|c|c|c|c|}
\hline \multirow{3}{*}{ Component } & \multicolumn{6}{|c|}{ Calibration $(n=308)$} & \multicolumn{6}{|c|}{ Test $(n=857)$} \\
\hline & \multicolumn{4}{|c|}{ Basic statistics (\% wt/wt) } & \multicolumn{2}{|c|}{ Pearson corr. } & \multicolumn{4}{|c|}{ Basic statistics (\% wt/wt) } & \multicolumn{2}{|c|}{ Pearson corr. } \\
\hline & Mean & SD & Min & Max & Fat & Protein & Mean & SD & Min & Max & Fat & Protein \\
\hline Fat & 3.44 & 0.86 & 1.61 & 6.25 & 1 & - & 3.54 & 0.79 & 1.54 & 5.72 & 1 & - \\
\hline Protein & 3.45 & 0.35 & 2.65 & 4.24 & 0.53 & 1 & 3.42 & 0.34 & 2.63 & 4.34 & 0.57 & 1 \\
\hline Lactose & 4.74 & 0.15 & 4.17 & 5.1 & -0.15 & -0.11 & 4.68 & 0.15 & 3.98 & 5.02 & -0.16 & -0.18 \\
\hline
\end{tabular}

375

376 Identically to the post-hoc models, the selected time interval corresponded to taking a new spectral 377 reference pair at the start of each session after connecting the sensor system to the AMS and every 0.5 378 hours after this initialization.

379 The prediction performances of the post-hoc and real-time approaches were mutually compared based 380 on the residuals for the samples in the test set (Table 4). Since the test sets for those two approaches were not identical, for each milk component the two approaches were compared with an unpaired t-test, in order to assess if there was a significant difference $(a=0.05)$ between them. The test results indicate that 383 the post-hoc models had a better performance for the prediction of all three milk components. 


\begin{tabular}{|c|c|c|c|c|c|c|}
\hline Component & Model & Preprocessing $^{\circ}$ & $\begin{array}{l}\text { Variable } \\
\text { Selection }\end{array}$ & LV & $\begin{array}{c}\text { RMSEP }^{\Delta}(\% \\
w t / w t)\end{array}$ & $R_{\mathrm{P}}^{2}$ \\
\hline \multirow[t]{2}{*}{ Fat } & Post-hoc & Detr SG2D(19) OSC & RiPLS & 3 & $0.078^{a}$ & 0.989 \\
\hline & Real-time & Detr SG2D(15) & FiPLS & 4 & $0.083^{b}$ & 0.989 \\
\hline \multirow[t]{2}{*}{ Protein } & Post-hoc & SNV SG1D(15) OSC & FiPLS & 10 & $0.08^{b}$ & 0.947 \\
\hline & Real-time & Detr SG2D(13) OSC & RiPLS & 5 & $0.11^{\mathrm{a}}$ & 0.894 \\
\hline \multirow[t]{2}{*}{ Lactose } & Post-hoc & MSC SG1D(19) & RiPLS & 16 & $0.077^{b}$ & 0.689 \\
\hline & Real-time & Log Base SG1D(07) OSC & RiPLS & 13 & $0.092^{\mathrm{a}}$ & 0.644 \\
\hline
\end{tabular}

${ }^{\circ}$ Mean centering is applied in all cases. Detr = detrend correction; $\operatorname{SG1D}(x)$ and $\operatorname{SG} 2 \mathrm{D}(x)=$ first and second Savitzky-Golay

386 derivatives with second polynomial order and with a window size of $x \mathrm{~nm}$; OSC = orthogonal signal correction; SNV = standard

387 normal variates; MSC = multiplicative scatter correction; Log = logarithmic spectral transformation; Base = baseline correction.

388 *FiPLS and RiPLS = forward and reverse interval partial least squares.

$389 \mathrm{LV}=$ number of latent variables selected; RMSEP = Root-mean-square error of prediction; $R_{\mathrm{P}}^{2}=$ coefficient of determination.

$390 \Delta^{\Delta}$ For each component, RMSEP-values with different superscripts indicate significant $(\alpha=0.05)$ differences between the different

391 models according to an unpaired t-test, with a letter lower in the alphabetical order indicating the best model.

393 The better performance of the post-hoc models compared to the real-time models was hypothesized, as

394 the former models were trained on samples selected over the entire experimental period of 8 weeks, thus

395 better representing the variability present in the samples of the test set. Moreover, the fatty acid or amino

396 acid composition of milk can drift over time due to an alteration in the diet or due to a change in the

397 lactation stage of the cows. Additionally, light scattering can be affected by a shift in the fat globule or

398 casein micelle size or by an alteration of their refractive index. Finally, also the environmental temperature

399 can affect the sample temperature. If these properties change, they affect the measured LW-NIR spectra

400 and thus the prediction performance (Aernouts et al., 2015a). 

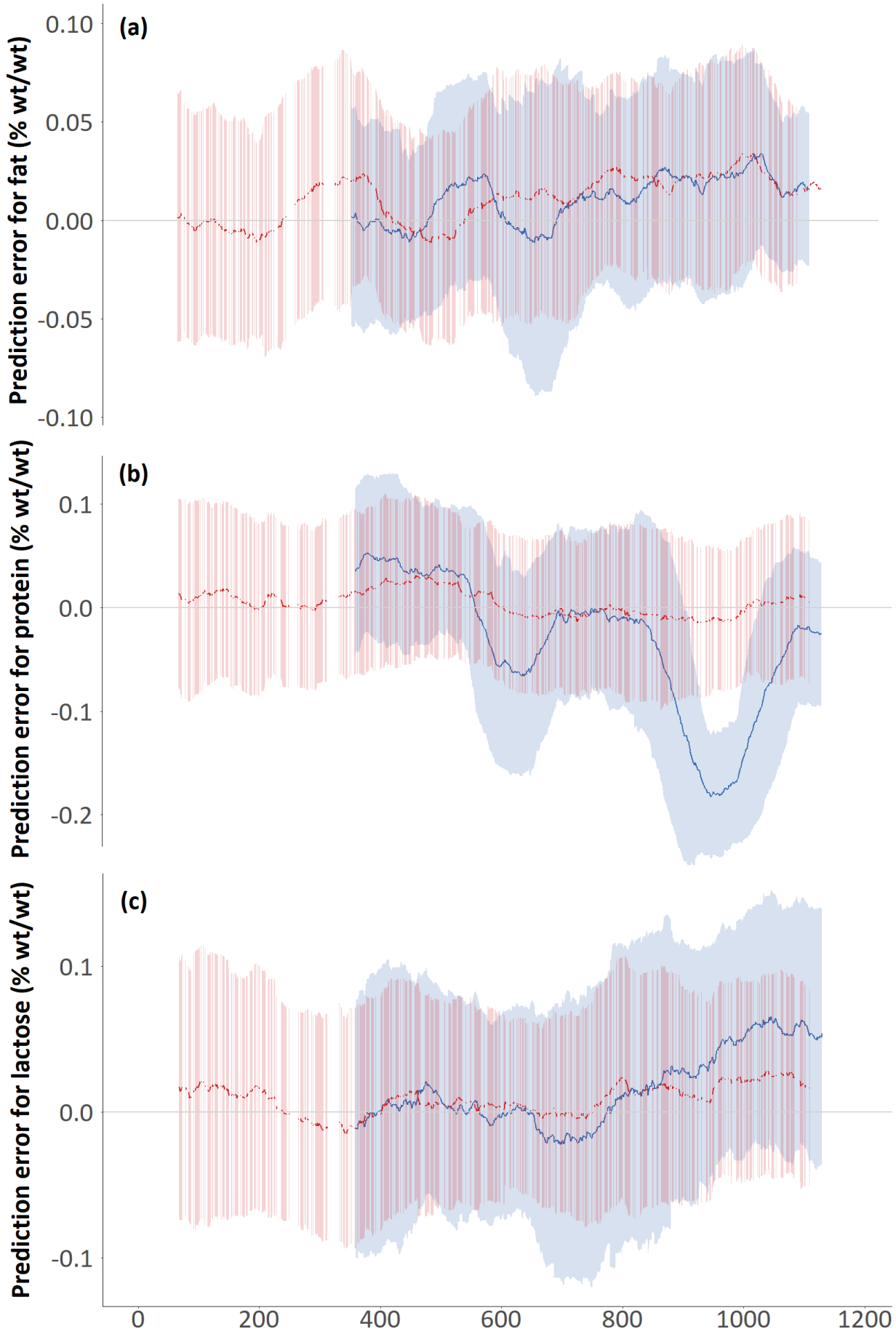

Figure 4. Prediction error plot for (a) fat, (b) protein, and (c) lactose, for the post-hoc (red) and real-time (blue) models, for milk

403 samples of the test set that are ordered based on the time when they were measured. A moving average (101 points) has been applied 404 to obtain the average prediction error (bold line) and the moving standard deviation (red and blue zones, for the post-hoc and real405 time models, respectively). The samples selected for calibration are not plotted (white zones). 
Figure 4 illustrates the drift observed in the prediction errors of (a) fat, (b) protein, and (c) lactose for the 407 test samples by the post-hoc (red) and real-time models (blue). The samples are sorted according to the 408 time when they were measured and they are synchronized for the different models. Because a moving 409 window of 101 points was used to calculate and illustrate the average prediction error and standard 410 deviation over time, with the increase of sample number, these parameters could not be shown for the 411 first and the last 50 samples of the test set. For the post-hoc models, the samples to train the PLSR models 412 are selected over the entire dataset following the duplex approach. Accordingly, the average prediction 413 error and standard deviation of the samples in the test set are interrupted with white zones representing 414 the samples used for calibration. For the real-time models, on the other hand, the calibration samples are 415 all taken at the beginning of the dataset (first week), appearing as a distinct white zone, and thus the test 416 set is a single uninterrupted series of samples.

417 For protein and lactose, the moving average prediction error of the post-hoc models remains close to zero, 418 with almost no trend with the increasing sample number. In contrast, milk fat prediction for the post-hoc 419 approach and all milk components of the real-time approach display drift over time. The real-time model 420 for the prediction of milk lactose shows a clear drift after week 6 (samples 845 - 1165) with mainly positive 421 prediction errors, indicating an underestimation of the lactose content by the model. The real-time model 422 to predict the milk protein shows apparent drift over the entire test set, although not being very 423 consistent. In weeks 3 and 4 (samples 539 to 677) and 7 (samples 941 - 1108), the prediction errors were 424 mainly negative, indicating an overestimation of the actual milk protein concentration by the models, 425 while they were mainly positive in week 2 (samples 349 - 538). The real-time model for the prediction of 426 milk fat resulted in mainly positive prediction errors in week 3 (sample 539 - 617) and after week 5 (sample 427678 - 1165), while the post-hoc counterpart resulted in mainly positive prediction errors in week 2 428 (samples 349 - 538) and after week 4 (samples 618 - 1165). All real-time models show an increased 429 standard deviation compared to their post-hoc counterparts, and this effect is larger for lactose. 
430 As the post-hoc models include calibration samples throughout the experiment, possible changes over 431 time in milk composition, environmental fluctuations, variations in the morphology and optical properties 432 of fat globules and casein micelles are, in general, more included in the calibration of the model. For fat, 433 we hypothesize that the greater sensitivity of this component to the external variations previously 434 mentioned might have a larger impact on the spectra than the variation in protein or lactose. In general, 435 this sampling procedure contributes to making this approach more robust against the observed drift. In 436 contrast, the real-time models display a more evident degradation of their prediction capabilities over 437 time. As these models are exclusively trained on the samples collected in the first week, they may not have 438 captured this additional complexity.

439 Table 4 and Figure 4 indicate that the variability of fat, protein, and lactose over this 8-week experiment 440 is not entirely captured by the calibration dataset of the real-time approach. This difference in milk 441 composition prediction between the two scenarios suggests that especially the real-time models can 442 benefit from calibration maintenance strategies to assure that the composition estimates remain accurate 443 under varying conditions. As such, changes due to management, parity, or diet that influence the 444 prediction performance could be included. By adding new samples to the calibration set that represent 445 this fluctuation, the models can be made robust against new measurement conditions, resulting in better 446 overall predictions (Wise and Roginski, 2015). The addition of new samples to the calibration set would 447 require the acquisition of additional reference analyses over time. Currently, individual cow milk samples 448 are already collected every 4 to 6 weeks as a part of milk recording programs. In the context of these 449 programs, the milk composition sensor system would increase the monitoring frequency of fat, protein, 450 and lactose, decreasing the need for milk composition reference analysis and its costs. The calibration 451 maintenance could still benefit from these existing programs by requesting the reference analysis of the 452 most informative samples to contribute to the calibration model. In addition, the milk composition 453 prediction could benefit from the implementation of spectral standardization methods (Bonfatti et al., 
create common NIR spectral databases from independent implementations of this sensor system in

456 different farms, or for different groups in the same farm.

457 The accuracy of the real-time prediction approach (Table 4), which includes the same cows in the 458 calibration and test set, was evaluated against the ICAR requirements for on-farm in-line and at-line milk 459 analyzers (ICAR, 2017b) and laboratory milk analyzers (ICAR, 2020). Good predictions were achieved for 460 protein, with an RMSEP value close to $0.11 \%$ (all \% are wt/wt), attaining sufficient accuracy for protein 461 predictions according to ICAR recommendations for at-line $(0.2 \%)$ and in-line $(0.25 \%)$ milk analyses (ICAR, 462 2017b). Very good results were obtained for fat and lactose, with RMSEP values under $0.1 \%$, accomplishing 463 the more restrictive accuracy limit requested for laboratory milk analyses (0.1\%).

464 The impact of drift related to environmental temperature, diet changes, lactation stage, etc. should be 465 further investigated in a study covering also a longer time period. Parallel measurement of these external 466 factors could help to better understand, distinguish between and even correct for different sources of 467 drift. Moreover, temperature monitoring and control could be applied to the milk sample, reducing the 468 effect of temperature variation in the spectral analysis.

4693.4 Analysis of the cow-specific bias on the milk predictions by the selected real-time model

470 The relationship between the median residuals of the calibration data and the prediction residuals of the 471 test data for the real-time prediction model are illustrated in Figure 5 for (a) fat, (b) protein, and (c) lactose 472 for each dairy cow. These plots evaluate if the cow-specific bias for each individual cow remains constant 473 between the calibration and test sets. The concordance correlation coefficient indicates practically no 474 correlation for fat and protein, but a weak relationship for lactose. 
(a)

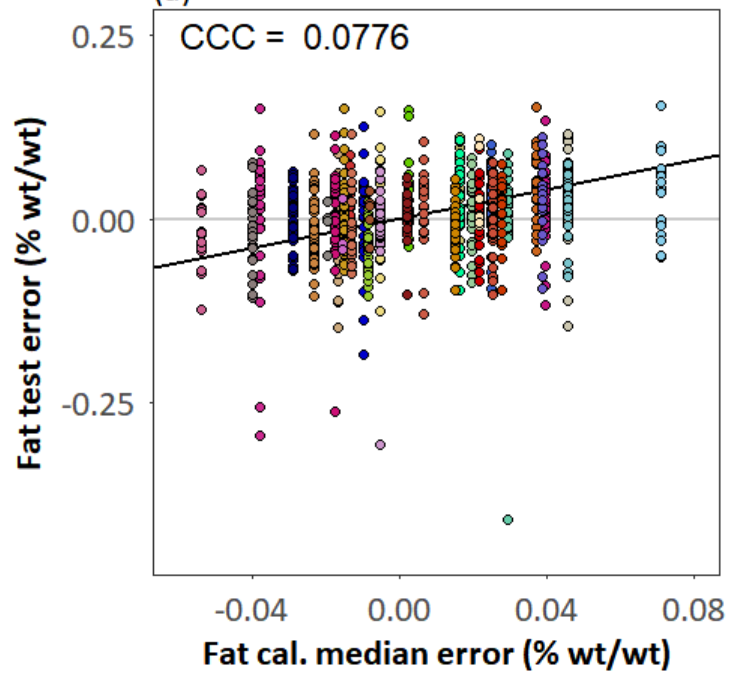

(b)

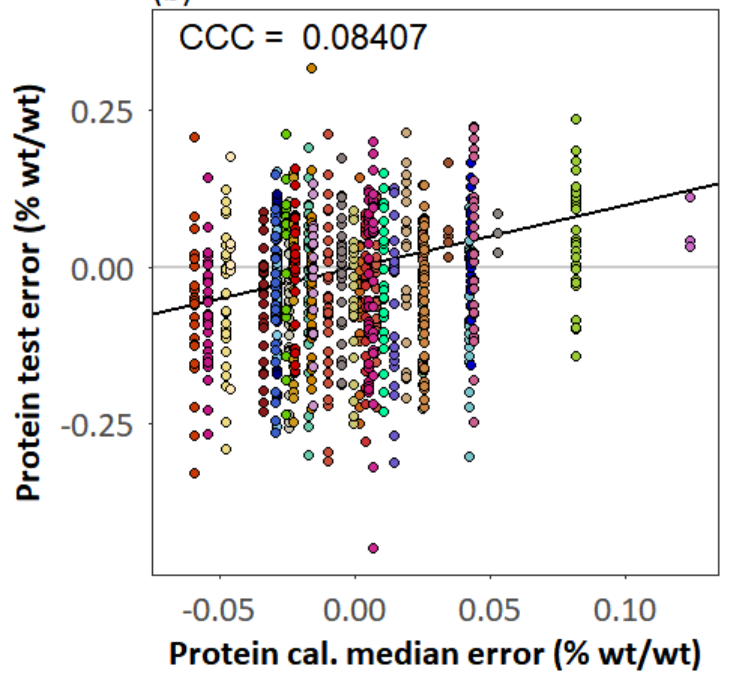

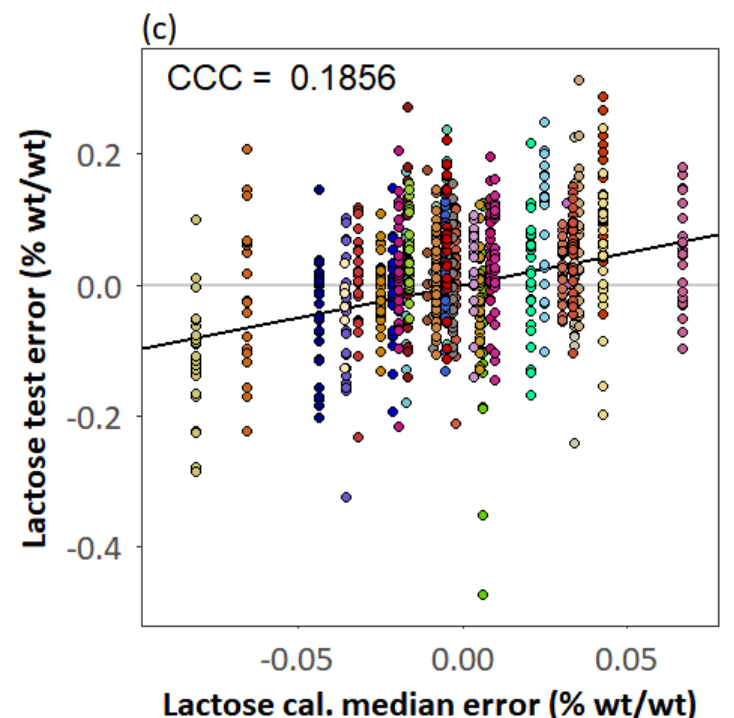

477 Figure 5. Relationship between prediction residuals and the medians of the calibration residuals for the real-time model, for every

478 individual dairy cow for (a) fat, (b) protein, and (c) lactose. Each color represents the predictions for a single cow. The black solid

479 lines are the identity lines. Three extreme fat test errors were outside the range of figure (a) fat (fat test error of -1.573, -0.661 and

$480-0.556 ;(\% w t / w t)) . C C C=$ concordance correlation coefficient.

481 Cow-specific bias correction was performed by subtracting the median residuals of the calibration data for 482 a single cow from all further milk composition predictions in the test set for that same animal. Next, the 483 prediction outcome of the original real-time model and the outcome after applying this cow-specific bias 
0.05) between them.

Table 5. Prediction performance statistics for the real-time and cow-specific bias correction prediction results.

\begin{tabular}{lcccccccc}
\hline \multirow{2}{*}{ Model } & \multicolumn{3}{c}{$\operatorname{RMSEP}^{\Delta}(\% \mathrm{wt} / \mathrm{wt})$} & & \multicolumn{3}{c}{$R_{\mathrm{P}}^{2}$} \\
\cline { 2 - 4 } \cline { 7 - 8 } & Fat & Protein & Lactose & & Fat & Protein & Lactose \\
\hline Real-time - No correction & 0.083 & 0.11 & $0.091^{\mathrm{b}}$ & & 0.989 & 0.894 & 0.644 \\
Real-time - Cow-specific bias correction & 0.084 & 0.109 & $0.088^{\mathrm{a}}$ & & 0.989 & 0.896 & 0.675
\end{tabular}

RMSEP = Root-mean-square error of prediction; $R_{\mathrm{P}}^{2}=$ coefficient of determination.

$488 \Delta^{\Delta}$ Within each column, RMSEP-values with different superscripts indicate significant $(\alpha=0.05)$ differences between the different models according to a paired t-test, with a letter lower in the alphabetical order indicating the best model.

The cow-specific bias correction resulted in a small improvement in the protein and lactose prediction, between the cow-specific bias correction included in the calibration and the cow specific bias over time. The RMSEP reduction on lactose increases its performance and brings it closer to the one of the post-hoc model for this milk component (RMSEP of $0.077 \%$ wt/wt and an $R_{\mathrm{P}}^{2}$ of 0.689 ) and further proves its suitability as a milk composition predictor (ICAR, 2017b \& 2020).

Three extreme fat test errors of negative sign (not shown in figure 5), with an absolute value greater than ten times the interquartile range, correspond to three different cows with median errors of positive sign in the calibration set. As the bias correction was performed by subtracting the median residuals of the calibration data from the predictions in the test set, the cow specific correction slightly increased the overall prediction error for fat for all samples. This correction results in a slightly decreased performance compared to not applying any correction for this milk component (Table 6). However, if the sample with

503 the most extreme fat test error (with an absolute value greater than 17 times the interquartile range) is 504 excluded, a very high fat prediction performance (RMSEP of $0.063 \% w t / w t$ and a $R_{\mathrm{P}}^{2}$ of 0.994 ) is obtained 
for the real-time approach, indicating that an improved overall milk fat prediction can be accomplished.

506 In this new scenario, the cow-specific bias correction for fat further improves (not significantly) the 507 prediction performance, given the newly increased relationship between prediction residuals and the 508 medians of the calibration residuals. However, the NIR spectrum and reference analysis results of this 509 sample were not outlying and no observations made during the trial pointed at valid reasons to justify the 510 removal of this sample. Therefore, this sample was kept in the analysis.

511 Cow-specific bias correction based on the subtraction of calibration median residuals from the predictions 512 in the test set provided a small, but significant performance increase on the real-time approach for lactose. 513 Therefore, further research is recommended on the application of different correction approaches that 514 more closely reflect the relationship between calibration and test data, since the cow-specific bias did not 515 remain constant over time, especially not for fat and protein. Cow-specific bias correction could further 516 improve when integrated with the aforementioned calibration maintenance procedures. However, the 517 complexity and cost of introducing reference acquisitions for all cows and potentially all lactations to 518 estimate the cow-specific correction has to be considered against the performance increase that the 519 correction can provide.

520 Finally, future tests should be performed closer to a real field application, with independent cow and herd 521 validations for the real-time approach.

\section{Conclusions}

523 An on-farm sensor system for online fat, protein, and lactose analysis was developed and implemented on

524 an AMS. This system can analyze the milk of each individual milking session autonomously to support the 525 cost-efficient monitoring of performance, efficiency, and welfare of individual dairy cows. The prediction 526 accuracy of the system was very high for all three milk components $(0.078 \%(w t / w t), 0.080 \%(w t / w t)$ and $527 \quad 0.077 \%(w t / w t)$ for fat, protein, and lactose respectively) when creating a prediction model following a 
post-hoc approach for sample selection. When implemented as a real-time system, training the prediction

529 model with the samples from the first week of the trial and evaluating the model performance on the

530 samples that were measured afterward, the prediction accuracies deteriorated to $0.083 \%$ (wt/wt), $0.110 \%$

531 (wt/wt) and 0.092\% (wt/wt) for fat, protein, and lactose, respectively. These performances still abide by

532 the ICAR guidelines for on-farm analysis systems for all components and even comply with the ICAR

533 thresholds for laboratory analysis systems for fat and lactose. Furthermore, the performance of the real-

534 time model for lactose prediction was further improved with the use of a cow-specific bias correction. As

535 drift was identified in the milk composition predictions over time, future research should focus on the

536 development and implementation of intelligent and efficient calibration maintenance procedures to cope

537 with external influences and guarantee long-term prediction stability.

\section{$538 \quad 5 \quad$ Acknowledgments}

539 This work was supported by the government agency Flanders Innovation and Entrepreneurship (VLAIO,

540 Belgium) through projects "Koesensor" (LA110770) and MastiMan (HBC.2016.0774). Milk samples were

541 collected within a trial set-up by ILVO for the "SMART melken" project (LA135081), funded by VLAIO. José

542 A. Diaz Olivares received funding from the Research Foundation Flanders (FWO, Belgium) through grant

543 No. $1576320 N$. Ines Adriaens received funding from a KU Leuven (Belgium) postdoctoral mandate grant

544 No. PDM/19/132.

\section{References}

547 Abd El -Fattah, A.M., Abd Rabo, F.H., EL-Dieb, S.M., Elkashef, H.A.S., 2012. Changes in composition of

$548 \quad$ colostrum of Egyptian buffaloes and Holstein cows. BMC Vet. Res. 8, 19.

$549 \quad$ https://doi.org/10.1186/1746-6148-8-19 
Aernouts, B., Adriaens, I., Diaz-Olivares, J., Saeys, W., Mäntysaari, P., Kokkonen, T., Mehtiö, T., Kajava, S., Lidauer, P., Lidauer, M.H., Pastell, M., 2019. Mid-Infrared Spectroscopic Analysis of Raw Milk to Predict the Blood Plasma Non-Esterified Fatty Acid Concentration in Dairy Cows. bioRxiv 853127. https://doi.org/10.1101/853127

Aernouts, B., Polshin, E., Lammertyn, J., Saeys, W., 2011. Visible and near-infrared spectroscopic analysis of raw milk for cow health monitoring: Reflectance or transmittance? J. Dairy Sci. 94, 5315-5329. https://doi.org/10.3168/jds.2011-4354

Aernouts, B., Van Beers, R., Watté, R., Huybrechts, T., Jordens, J., Vermeulen, D., Van Gerven, T., Lammertyn, J., Saeys, W., 2015a. Effect of ultrasonic homogenization on the Vis/NIR bulk optical properties of milk. Colloids Surfaces B Biointerfaces 126, 510-519. https://doi.org/10.1016/j.colsurfb.2015.01.004

Aernouts, B., Van Beers, R., Watté, R., Huybrechts, T., Lammertyn, J., Saeys, W., 2015b. Visible and nearinfrared bulk optical properties of raw milk. J. Dairy Sci. 98, 6727-6738. https://doi.org/10.3168/jds.2015-9630

Andersen, H.V., Wedelsback, H., Hansen, P.W., 2013. A white paper from FOSS: NIR spectrometer technology comparison. FOSS P/N 1026672, 1-14.

Anderson, G.P.S., Zhang, I.L., Winkelman, A.M., Harris, B.L., 2017. Comparison of records from in-line milk meters and conventional herd testing for management and genetic evaluation of dairy cows, in: ICAR Technical Series. pp. 203-209.

Bobbo, T., Cipolat-Gotet, C., Bittante, G., Cecchinato, A., 2016. The nonlinear effect of somatic cell count on milk composition, coagulation properties, curd firmness modeling, cheese yield, and curd nutrient recovery. J. Dairy Sci. 99, 5104-5119. https://doi.org/10.3168/jds.2015-10512 
572 Bogomolov, A., Melenteva, A., 2013. Scatter-based quantitative spectroscopic analysis of milk fat and 573 total protein in the region $400-1100 \mathrm{~nm}$ in the presence of fat globule size variability. Chemom. 574 Intell. Lab. Syst. 126, 129-139. https://doi.org/10.1016/j.chemolab.2013.02.006

575 Brandt, M., Haeussermann, A., Hartung, E., 2010. Invited review: Technical solutions for analysis of milk 576 constituents and abnormal milk. J. Dairy Sci. https://doi.org/10.3168/jds.2009-2565

577 Cederkvist, H.R., Aastveit, A.H., Næs, T., 2005. A comparison of methods for testing differences in 578 predictive ability. J. Chemom. 19, 500-509. https://doi.org/10.1002/cem.956

579 Fadul-Pacheco, L., Lacroix, R., Séguin, M., Grisé, M., Vasseur, E., Lefebvre, D., 2018. Comparison of milk 580 composition and somatic cell count estimates from automatic milking systems sensors and milk 581 recording laboratory, in: Proceedings of the 42nd ICAR Conference. Auckland, New Zealand.

Forsbäck, L., Lindmark-Månsson, H., Andrén, A., Åkerstedt, M., Andrée, L., Svennersten-Sjaunja, K., 2010. Day-to-day variation in milk yield and milk composition at the udder-quarter level. J. Dairy Sci. 93,

Forsbäck, L., Lindmark-Månsson, H., Åndrén, A., Kerstedt, M., Svennersten-Sjaunja, K., 2009. Udder quarter milk composition at different levels of somatic cell count in cow composite milk. Animal 3,

Gonçalves, J.L., Tomazi, T., Barreiro, J.R., Beuron, D.C., Arcari, M.A., Lee, S.H.I., Martins, C.M. de M.R., Araújo Junior, J.P., Santos, M.V. dos, 2016. Effects of bovine subclinical mastitis caused by Corynebacterium spp. on somatic cell count, milk yield and composition by comparing contralateral quarters. Vet. J. 209, 87-92. https://doi.org/10.1016/j.tvjl.2015.08.009 Other Quantitative Calibration Methods and the Extraction of Qualitative Information. Anal. Chem. 
ICAR. 2017a. Section 11 - Guidelines for Testing, Approval and Checking of Milk Recording Devices. International Committee on Animal Recording, Rome, Italy.

ICAR. 2017b. Section 13 - Guidelines for On-Line Milk Analysis. International Committee on Animal Recording, Rome, Italy.

ICAR. 2020. Section 12 - Guidelines for Milk Analysis. International Committee on Animal Recording, Rome, Italy.

ISO, 2013. Milk and liquid milk products -- Guidelines for the application of mid-infrared spectrometry. Page 14 in International Standard ISO 9622:2013/IDF 141:2013. International Dairy Federation.

Kaniyamattam, K., De Vries, A., 2014. Agreement between milk fat, protein, and lactose observations collected from the Dairy Herd Improvement Association (DHIA) and a real-time milk analyzer. J. Dairy Sci. 97, 2896-2908. https://doi.org/10.3168/jds.2013-7690

Kawamura, S., Kawasaki, M., Nakatsuji, H., Natsuga, M., 2007. Near-infrared spectroscopic sensing system for online monitoring of milk quality during milking. Sens. Instrum. Food Qual. Saf. 1, 37-43.

Kemps, B.J., Saeys, W., Mertens, K., Darius, P., De Baerdemaeker, J.G., De Ketelaere, B., 2010. The importance of choosing the right validation strategy in inverse modelling. J. Near Infrared Spectrosc. 18, 231-237. https://doi.org/10.1255/jnirs.882 https://doi.org/10.1007/s11694-006-9001-x

Kawasaki, M., Kawamura, S., Tsukahara, M., Morita, S., Komiya, M., Natsuga, M., 2008. Near-infrared spectroscopic sensing system for on-line milk quality assessment in a milking robot. Comput. Electron. Agric. 63, 22-27. https://doi.org/10.1016/j.compag.2008.01.006

Lin, L.I.-K., 1989. A Concordance Correlation Coefficient to Evaluate Reproducibility. Biometrics 45, 255. 
Mäntysaari, P., Mäntysaari, E.A., Kokkonen, T., Mehtiö, T., Kajava, S., Grelet, C., Lidauer, P., Lidauer, M.H., 2019. Body and milk traits as indicators of dairy cow energy status in early lactation. J. Dairy Sci. 102, 7904-7916. https://doi.org/10.3168/jds.2018-15792

McParland, S., Lewis, E., Kennedy, E., Moore, S.G., McCarthy, B., O'Donovan, M., Butler, S.T., Pryce, J.E., Berry, D.P., 2014. Mid-infrared spectrometry of milk as a predictor of energy intake and efficiency in lactating dairy cows. J. Dairy Sci. 97, 5863-5871. https://doi.org/10.3168/jds.2014-8214

Melfsen, A., Hartung, E., Haeussermann, A., 2012. Accuracy of in-line milk composition analysis with

R Core Team, 2018. R: A language and environment for statistical computing. R Foundation for Statistical Postelmans, A., Aernouts, B., Jordens, J., Van Gerven, T., Saeys, W., 2020. Milk homogenization monitoring: Fat globule size estimation from scattering spectra of milk. Innov. Food Sci. Emerg. Technol. 60, 102311. https://doi.org/https://doi.org/10.1016/j.ifset.2020.102311 Computing, Vienna, Austria. URL https://www.R-project.org/.

Rosati, A., Van Vleck, L.D., 2002. Estimation of genetic parameters for milk, fat, protein and mozzarella cheese production for the Italian river buffalo Bubalus bubalis population. Livest. Prod. Sci. 74, 185190. https://doi.org/10.1016/S0301-6226(01)00293-7

Saeys, W., Beullens, K., Lammertyn, J., Ramon, H., Naes, T., 2008. Increasing robustness against changes in the interferent structure by incorporating prior information in the augmented classical leastsquares framework. Anal. Chem. 80, 4951-4959. https://doi.org/10.1021/ac800155n 
components and total aerobic bacteria count of raw milk. J. Near Infrared Spectrosc. 16, 389-398. https://doi.org/10.1255/jnirs.807

640

Shetty, N., Min, T.G., Gislum, R., Olesen, M.H., Boelt, B., 2011. Optimal sample size for predicting viability of cabbage and radish seeds based on near infrared spectra of single seeds. J. Near Infrared Spectrosc. 19, 451-461. https://doi.org/10.1255/jnirs.966

Slutsky, B., 1997. Handbook of Chemometrics and Qualimetrics: Part A, Data Handling in Science and Technology Volume 20A. Elsevier, Amsterdam. https://doi.org/10.1021/ci980427d

Snee, R.D., 1977. Validation of Regression Models: Methods and Examples. Technometrics 19, 415-428. https://doi.org/10.1080/00401706.1977.10489581

Tsenkova, R., Atanassova, S., Ozaki, Y., Toyoda, K., Itoh, K., 2001. Near-infrared spectroscopy for biomonitoring: Influence of somatic cell count on cow's milk composition analysis. Int. Dairy J. 11,

Tsenkova, R., Atanassova, S., Toyoda, K., Ozaki, Y., Itoh, K., Fearn, T., 1999. Near-infrared spectroscopy

Wise, B.M., Roginski, R.T., 2015. A calibration model maintenance roadmap, in: IFAC-PapersOnLine. pp. 2351. https://doi.org/10.3168/jds.S0022-0302(99)75484-6 Double integrating sphere measurements for estimating optical properties of pig subcutaneous adipose tissue. Innov. Food Sci. Emerg. Technol. 19, 218-226. 


\section{Declaration of interests}

૫ The authors declare that they have no known competing financial interests or personal relationships that could have appeared to influence the work reported in this paper.

$\square$ The authors declare the following financial interests/personal relationships which may be considered as potential competing interests:

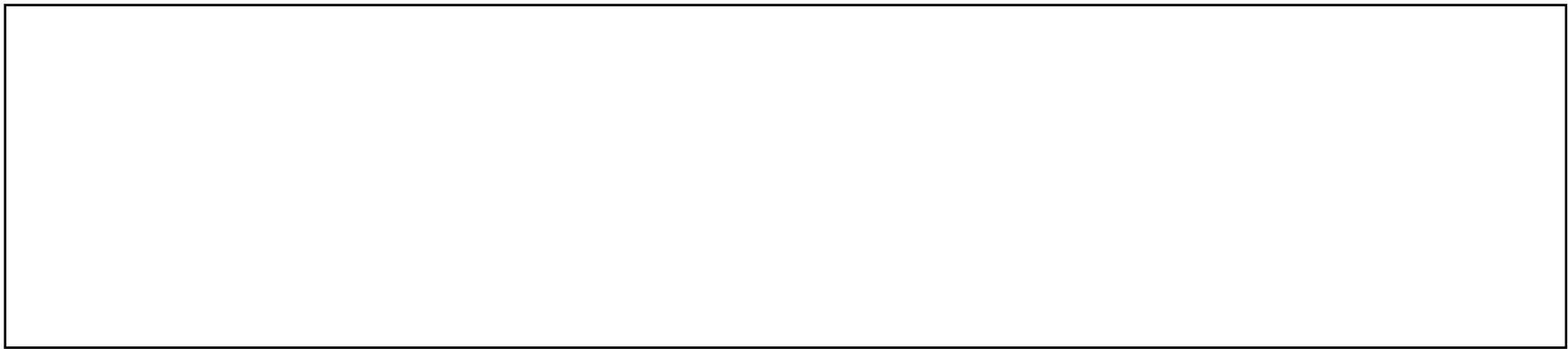




\section{CRediT authorship statement}

Jose A. Diaz-Olivares: Methodology, Software Programming, Validation, Formal Analysis, Data Curation, Writing - Original Draft, Review and Editing Preparation; Ines Adriaens: Formal Analysis, Data Curation, Writing - Review \& Editing; Els Stevens: Resources; Wouter Saeys: Conceptualization, Writing - Review \& Editing; Ben Aernouts: Conceptualization, Methodology, Investigation, Supervision, Project Acquisition, Writing - Review \& Editing, Project Administration, Funding Acquisition;

All authors have read and agreed to the published version of the manuscript. 